\title{
Advanced In-Situ Instrumentation of RF Circuits for Mixed-Field Irradiation Testing Purpose
}

\author{
Steffen Mueller, Salvatore Danzeca, Rubén García Alía, Markus Brugger, Robert Weigel, and Alexander Koelpin
}

\begin{abstract}
This paper presents a method that applies multiplexing and in-fixture calibration technique to qualify for efficient instrumentation of multiple $\mathrm{RF}$ circuits up to $6 \mathrm{GHz}$ during mixed-field irradiation.
\end{abstract}

Index Terms-In-situ test, automated test equipment, radio frequency, radiation effects.

\section{Motivation}

At high-energy irradiation facilities equipment is typically located several tens of meters away from the device under test (DUT). This poses a challenge to test bed design, especially for the investigation of radio frequency (RF) circuits. Setups are prone to systematic errors and parameter drifts, leading to measurement uncertainty, misinterpretation of results, or even masking of radiation-induced effects. Re-calibration of equipment by operator interaction is usually not possible at runtime. Furthermore, RF measurements require special equipment such as vector network analyzer (VNA) or spectrum analyzer. These devices are typically not able to measure more than one DUT at a time. In order to comply with requirements of relevant testing standards [1], [2], [3], [4], multiple DUTs are required to be tested to achieve reasonable significance of results. This paper proposes a method for accurate and efficient instrumentation of multiple DUTs. The concept qualifies for automated measurements of RF circuits during irradiation. Test bed performance is optimized for $2.4 \mathrm{GHz}$ applications, whereas the hardware is basically applicable up to $6 \mathrm{GHz}$.

\section{TEST BED CONCEPT}

\section{A. Overview CHARM Facility}

CHARM is considered as mixed-field radiation facility that is situated at the Proton Synchrotron (PS) complex at the European Organization for Nuclear Research (CERN). Fig. 1 gives an overview about the conceptual layout of the irradiation area. A $24 \mathrm{GeV}$ proton beam is extracted from PS and impinges on a metal target in order to generate a mixedfield of secondaries. By applying different metal targets and variable shielding configurations, a variety of representative

S. Mueller and R. Weigel are with the Institute for Electronics Engineering (LTE) at the Friedrich-Alexander University ErlangenNuremberg (FAU), Cauerstr. 9, 91058 Erlangen, Germany. E-mail: \{ste.mueller | robert.weigel $\} @$ fau.de

S. Danzeca, R. García Alía, and M. Brugger are with the European Organization for Nuclear Research (CERN), CH-1211 Geneve 23, Switzerland. E-mail: \{salvatore.danzeca | ruben.garcia.alia I markus.brugger\}@ cern.ch

A. Koelpin is with the Chair for Electronics and Sensor Systems at the Brandenburg University of Technology Cottbus-Senftenberg (BTU), SiemensHalske-Ring 14, 03046 Cottbus, Germany. E-mail: alexander.koelpin@b-tu.de

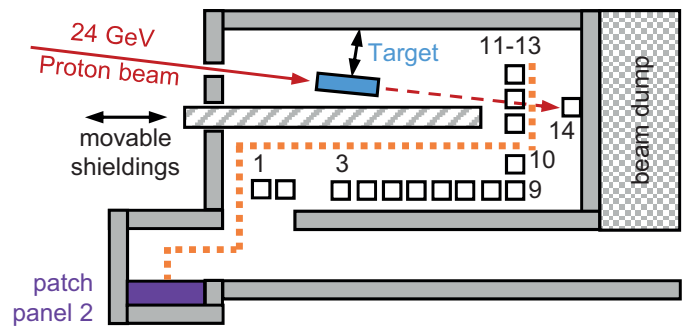

Figure 1. Conceptual layout of the irradiation area at CHARM facility.

radiation spectra can be emulated. A detailed description of the facility is found in [5]. Automated testing equipment (ATE) is located in the control room during irradiation phase. Signals from the ATE are connected to patch panel 1 inside the control room. Static cabling connects patch panel 1 with patch panel 2 (violet) inside the irradiation area. Movable cabling bridges the distance between patch panel 2 and the final DUT position (orange dots). These cables are encapsulated in support chains which are suspended from the ceiling and pulled behind when the DUT is automatically brought to the test positions 1 to 14 by robotic equipment. The total one-way cable length calculates to $45 \mathrm{~m}$. Fig. 2 shows the simplified connection topology and corresponding distances for cabling at CHARM facility.

\section{B. Test Bed Topology}

Fig. 3 depicts the proposed test bed topology for instrumentation of four DUTs. It is important to note that green colored parts reside in non-radiated areas, whereas yellow colored parts stay within the radiation area. The stimulus signal is injected from port 1 of the VNA and passes an optional preamplifier that provides additional signal boosting to compensate for downlink losses. A subsequent 2-way multiplexer (1:2 MUX) branches the signal either to a power sensor for source power calibration, or straight into patch panel 1 towards the

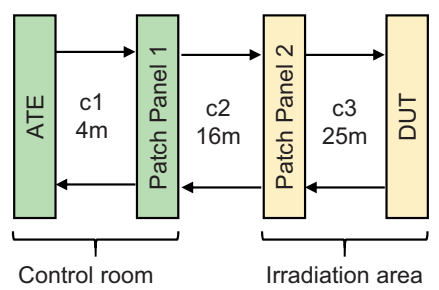

Figure 2. Simplified connection topology and distances at CHARM facility. 


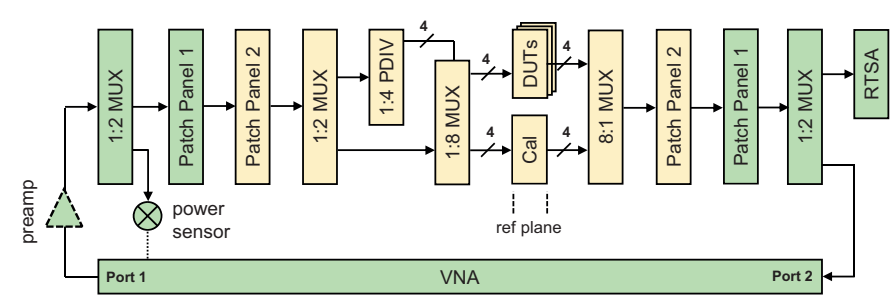

Figure 3. Topology of RF paths for in-situ instrumentation of four DUTs.

irradiation area. Inside the irradiation area the signal forks right in front of the DUT using another 2-way multiplexer. Then it feds either into a 4-way power divider (PDIV) or into an 8-way multiplexer (1:8 MUX). The power divider is used for equal biasing of DUTs when no measurements are performed by the VNA, whereas the 8-way multiplexer is allows to connect port 1 of the VNA selectively to a specific DUT, or alternatively to the calibration standards (CAL). The uplink path from the DUT to the control room is essentially similar to the downlink path and even less complex. At control room area an 2-way multiplexer connects the DUTs either to the VNA or to a real-time spectrum analyzer (RTSA). The latter enables for triggering on unintended transient spectral content in the DUT's output signal.

\section{Multiplexing}

A key advantage of the proposed topology is the concept of multiplexing. Classical 1-by-1 instrumentation requires $N$ signal sources and $N$ sinks for the instrumentation of $N$ DUTs (Fig. 4a), resulting in huge effort and high costs for test bed installation. This approach also introduces measurement uncertainty due to both individual calibration of measurement devices, and independent drift of test bed equipment, making post-processing and interpretation of results difficult. Instead, the concept of multiplexing greatly simplifies the instrumentation topology (Fig. 4b). Signals are generated and acquired using only one source and sink for stimulus and acquisition respectively. Thus, calibration and costs reduce significantly. Moreover, downlink and uplink cabling can be shared to overcome variations of single cable paths. Furthermore, reference plane calibration can be conveniently integrated by

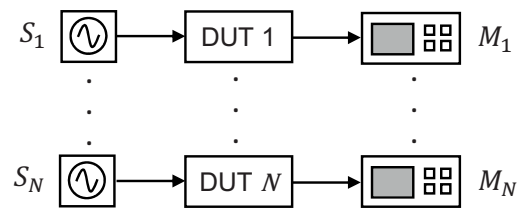

(a) 1-by-1 instrumentation

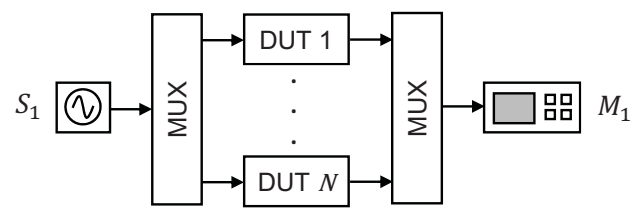

(b) 1-by-N instrumentation

Figure 4. Comparison of different instrumentation approaches.

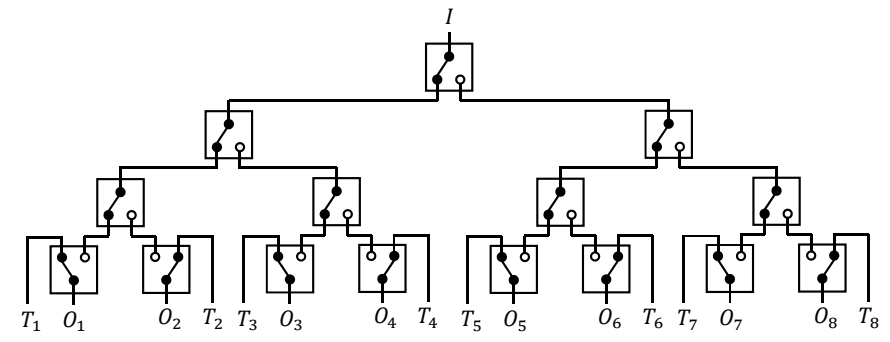

(a) Switching architecture.

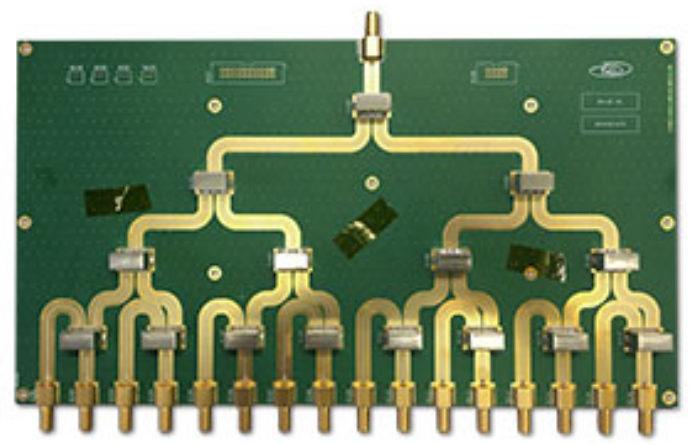

(b) Printed circuit board.

Figure 5. Architecture and realization of the 1:8 RF multiplexer.

applying calibration standards in parallel to the DUTs. In order to achieve maximum benefit from multiplexing, the multiplexers are required to be located as close as possible to the DUTs, that means they have to stay inside the radiation locale where they are exposed to radiation, too. Therefore, a custom designed radiation hardened 8-way multiplexer is carried out. Its architecture implements binary tree signal routing using single-pole-single-throw electromechanical RF switch technology (Fig. 5a). These switches are preferred due to their inherent radiation hardness while providing high isolation, low insertion loss, good input/output matching, and high contact rating. The printed circuit board (PCB) features a strictly symmetric $50 \mathrm{Ohm}$ impedance controlled design on a dedicated RF substrate (Fig. 5b). The design is optimized for both, high bandwidth and low insertion loss. The outputs $O_{1}$ to $O_{4}$ are connected to the DUTs' inputs, whereas $O_{4}$ to $\mathrm{O}_{8}$ are attached to the calibration kit. During measurement phase the ATE selects the RF path to the desired DUT by controlling corresponding switches of the multiplexer. When no measurements are performed, all switches of the output stage are toggled to the termination position in order to make the DUTs' input accessible on the outputs $T_{1}$ to $T_{4}$. These outputs are either directly connected to the output of the 4-way power divider or equipped with $50 \mathrm{Ohm}$ terminations. Thereby, DUTs can be either operated with equally biased with RF signals or unbiased, depending on the goals of the radiation qualification procedure.

\section{RF Path Characteristics}

Fig. 6 depicts both the insertion loss and return loss of the downlink RF signal path from the VNA to the DUT. The insertion loss is $12.5 \mathrm{~dB}$ at $2.4 \mathrm{GHz}$. The calculation in (1) emphasizes the main contributions to the path attenuation at 

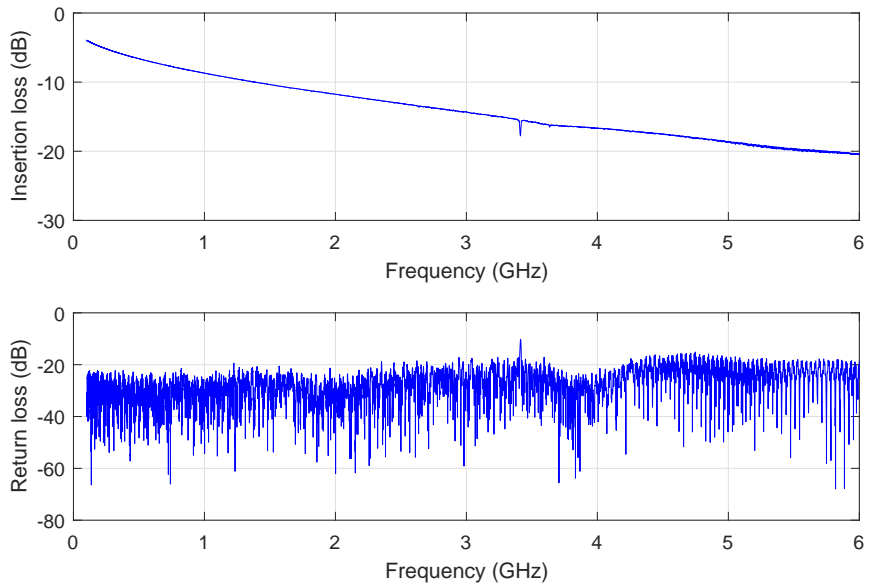

Figure 6. Insertion loss and return loss from ATE to the DUT's input.

$2.4 \mathrm{GHz}$. The total insertion loss $A_{\text {tot }}$ is determined by the attenuation of the downlink cables $A_{c 1}, A_{c 2}, A_{c 3}$, plus the insertion loss of the multiplexers.

$$
\begin{aligned}
A_{\mathrm{tot}} & \approx 2 A_{\mathrm{MUX} 1: 2}+A_{\mathrm{c} 1}+A_{\mathrm{c} 2}+A_{\mathrm{c} 3}+A_{\mathrm{MUX} 1: 8} \\
& =(2 \cdot 0.6+0.9+2.4+5.5+2.5) \mathrm{dB}=12.5 \mathrm{~dB}
\end{aligned}
$$

The return loss is less than $-20 \mathrm{~dB}$ up to $4.2 \mathrm{GHz}$ and about $-18 \mathrm{~dB}$ from $4.2 \mathrm{GHz}$ to $6 \mathrm{GHz}$. Taking into account that directivity of state-of-the-art VNAs is at least $50 \mathrm{~dB}$, the remaining headroom of about $25 \mathrm{~dB}$ provides good accuracy for return loss measurements at $2.4 \mathrm{GHz}$.

\section{E. Measurement Uncertainty}

The test bed may experience three categories or errors: systematic errors, drift errors, and random errors.

Systematic errors can be automatically removed from the measurement data by VNA calibration (see Section II-F). If systematic errors vary over time, cyclic re-calibration must be triggered by the ATE. Most prominent systematic error sources are: loss in RF cables and connectors, insertion loss of the multiplexer, and the imperfection of VNA device itself.

Drift errors may affect the RF measurement device or the test bed equipment. Especially amplitude and phase response of cable is altered when they are subject to either mechanical stress or change in ambient temperature. Temperature drift of cables was investigated using a laboratory mock-up. The test bed was configured for a closed loop setup, i. e. with no DUT in place, resulting in $90 \mathrm{~m}$ two-way cable length. Fig. 7 depicts the drift of both insertion phase and insertion loss for a runtime of $42 \mathrm{~h}$ with respect to room temperature changes of $\pm 2^{\circ} \mathrm{C}$. It can be seen that signals may experience a change of insertion phase up to $10 \mathrm{el}^{\circ}$, whereas change of insertion loss is relatively small. Hence re-calibration must be considered in particular for phase related measurement.

Random errors are not predictable and can not be completely removed but reduced to a certain extend. From instrumentation point of view noise is the most relevant error source. For optimum noise suppression, a trade-off between intermediate frequency (IF) bandwidth and measurement time
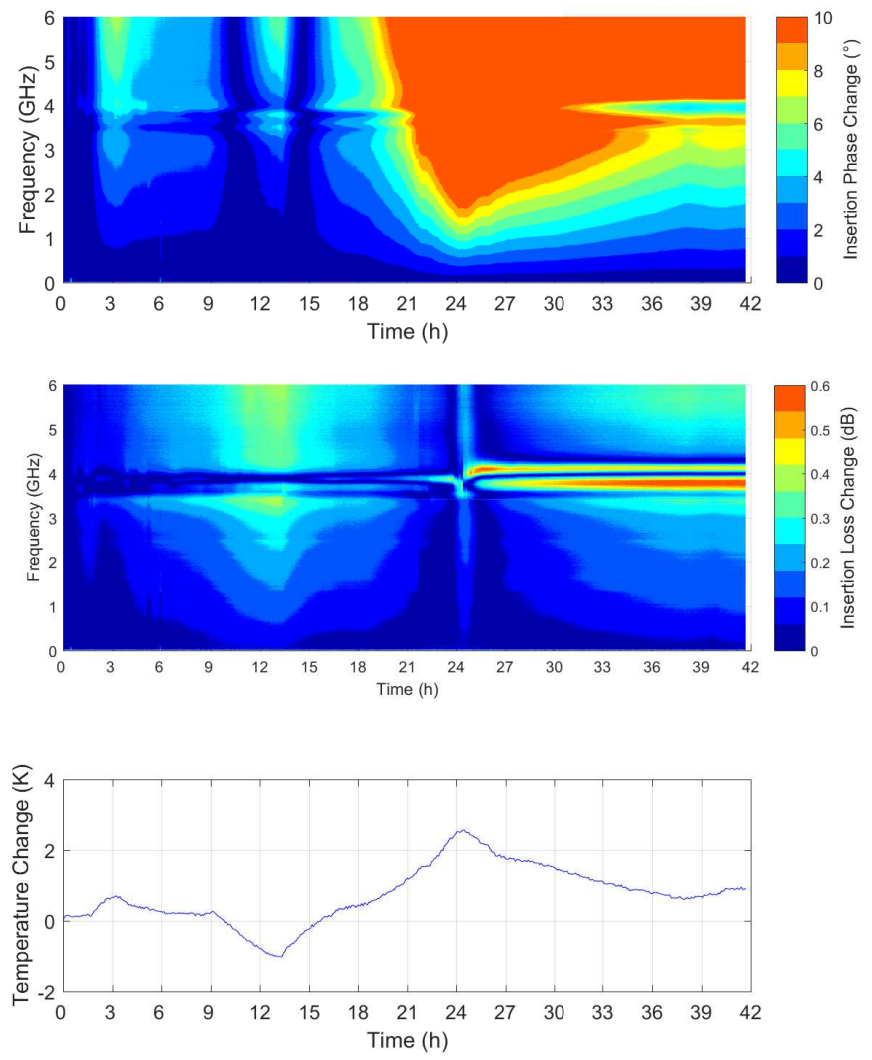

Figure 7. Example for drift of insertion loss and insertion phase of the cable setup with respect to change in ambient temperature.

has to be made. Narrowing the IF yields enhanced noise performance but results in increased in sweep time. The latter increases in turn the "dead time" of experiment, that means the time where no other measurements can be performed until the frequency sweep has finished.

\section{F. Calibration}

1) Vector error correction: Characteristics of RF circuits are usually expressed by so called scattering parameter ( $\mathrm{S}$ parameter) which are measures of relative wave quantities. The parasitic effects of the test bed setup overlay with the wanted S-parameter measurements. Modern VNAs apply vector error correction (VEC) in order to automatically remove the effects of the test bed from measurement results. Therefore, a calibration routine has to be performed. Thru-reflect-line (TRL) [6] is the preferred calibration standard for this setup, as it qualifies for in-fixture calibration where single calibration standards can be efficiently integrated on RF substrates together with the DUT circuit.

2) Power calibration: Certain measurement tasks (e.g. amplifier characterization) require the acquisition of absolute wave quantities. Power calibration refers to the action of characterization the VNA's imperfect internal RF signal source while considering losses in the test fixture in order to calculate precisely actual power levels at the DUT's input. Power calibration is performed using a power sensor device which is an active and highly sensitive device that is not suited for radiation exposure, thus it must reside in the control room 


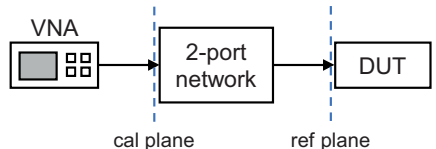

Figure 8. Calibration plane and reference plane for power calibration.

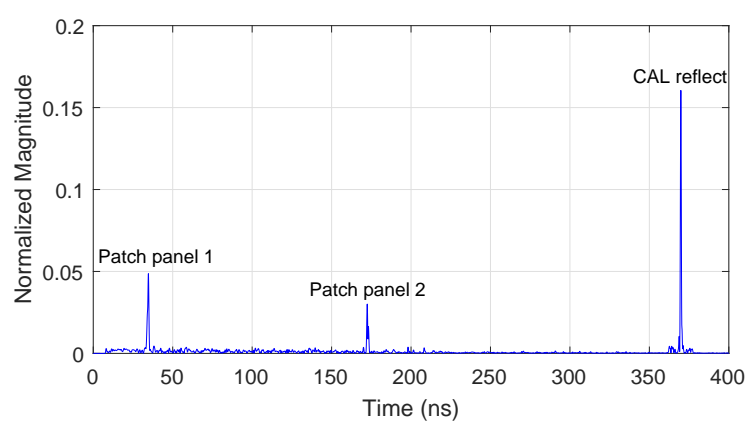

Figure 9. TDR representation of the downlink path from VNA to the reflection standard of the calibration kit.

(Fig. 3). However, the power calibration plane (cal plane) at the control room can be virtually shifted to the DUT's input reference pane (ref plane) by introducing a 2-port network in between the calibration plane and the DUT (Fig. 8), provided the forward transmission parameter $S_{21}$ of the downlink path is known. $S_{21}$ can be efficiently determined during runtime using time-domain reflectometry (TDR). Therefore, the 8-way multiplexer is switched to the reflection standard of the calibration kit. By applying gating to the time domain response of the single port reflection measurement (Fig.9) and converting data back to frequency domain, $S_{21}$ can be retrieved from $S_{11}$ measurement [7].

\section{EXPERIMENTAL RESULTS}

First experiments in radiation environment were conducted at CHARM facility using three $2.4 \mathrm{GHz}$ synthesizers as DUTs. The synthesizer circuit is a custom radiation tolerant boardlevel design which was carried out by the authors [8]. Since S-parameter measurements were not relevant during this test, the topology presented in Section II-B was slightly simplified by removing both calibration kit and the 8-way multiplexer in the downlink path. All DUTs were equally biased from a common $20 \mathrm{MHz}$ reference which was supplied via the 4-way power divider. Fig. 10a shows the hardware setup of the DUTs during the dry-run. The multiplexer, the power divider, and the power supply distribution were enclosed in 19" rack chassis in order to achieve a mechanically robust setup. DUTs were placed at rack position \#10 (Fig. 10b). In total 1587 parameter measurements were recorded for each DUT during the total experiment runtime of $147 \mathrm{~h}$. DUTs were irradiated for $96 \mathrm{~h}$ with aluminum target (al_0000) and 51h with copper target (cp_0000) configuration, yielding an $1 \mathrm{MeV}$ neutron equivalent fluence of $8 \cdot 10^{11} \mathrm{~cm}^{-2}$ and an accumulated dose of $112 \mathrm{~Gy}$.

Fig. 11 shows the relative change of output frequency over time. The standard deviation of output frequency change is about $6 \mathrm{~Hz}$, which is below the specified uncertainty of the measurement equipment $( \pm 10 \mathrm{~Hz})$. Obviously all DUTs

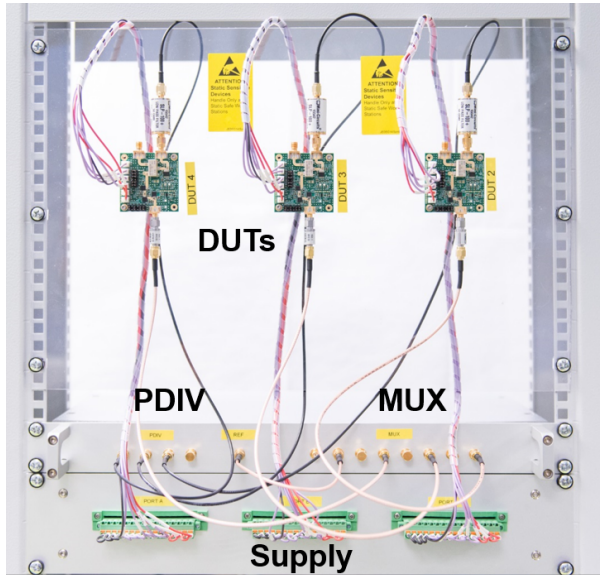

(a) Dry-run setup.

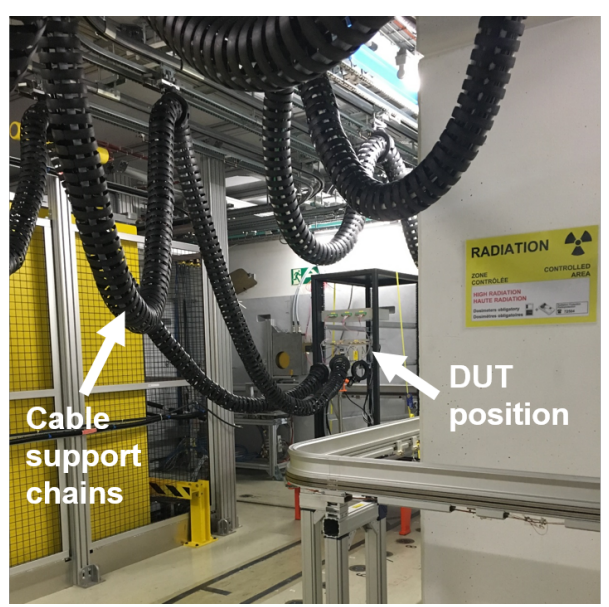

(b) Irradiation setup.

Figure 10. Hardware setup during dry-run and irradiation.

are closely matched over time. At the end of experiment, the change in output frequency is about $-10 \mathrm{~Hz}$ which is considerably small. But temporal drift of output frequency over time is clearly observable. It is found that both effects were not related to radiation, but correlate to the thermal drift of the common $20 \mathrm{MHz}$ reference source, which was externally supplied to from the ATE. It could be verified that the signal source experienced ambient temperature changes according to the temperature profile that was recorded in the control room.

Fig. 12 shows the change in output power of the $2.4 \mathrm{GHz}$ carrier over time. Again, DUT responses match close to each other. A slight increase in amplitude is observed, but changes stay below the uncertainty specification of the equipment $( \pm 0.6 \mathrm{dBm})$. However, there are two significant events where all curves noticeably drop. As all DUTs are simultaneously affected it is assumed that this was triggered by an unexpected systematic error. At least one of the two events was found to correlate to a loss of beam event. Since the signatures of both events are very similar, both notches are expected to have the same cause.

\section{CONCLUSION}

Multiplexing together with in-fixture calibration is a versatile, robust, and efficient approach for in-situ irradiation testing 

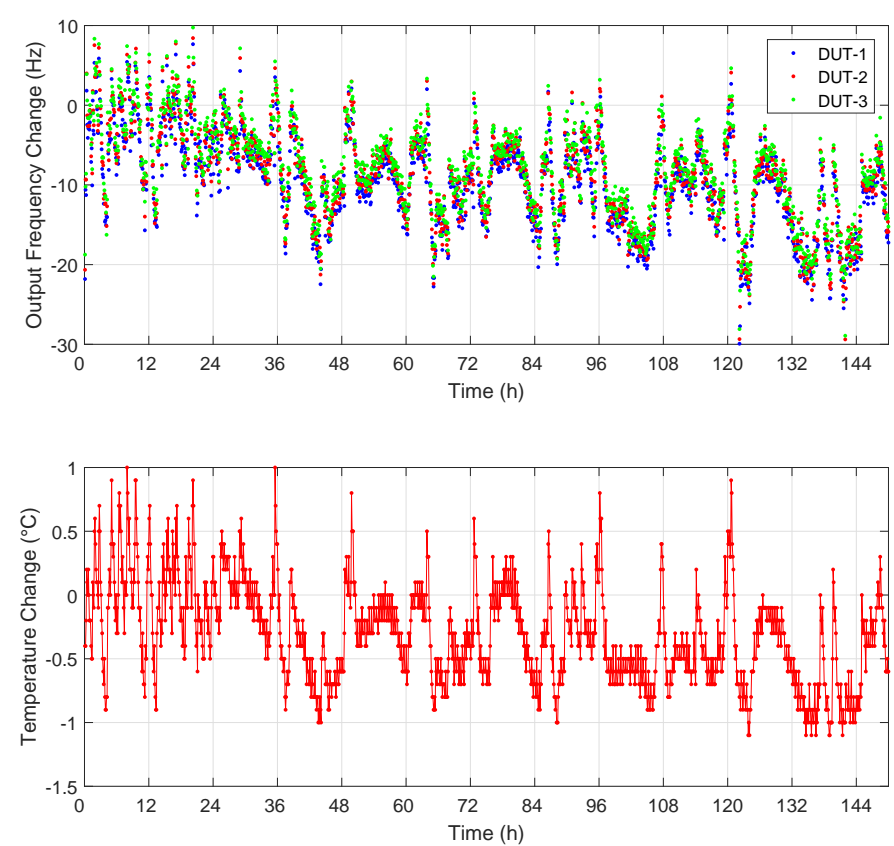

Figure 11. Change of output frequency over time w.r.t. temperature profile.

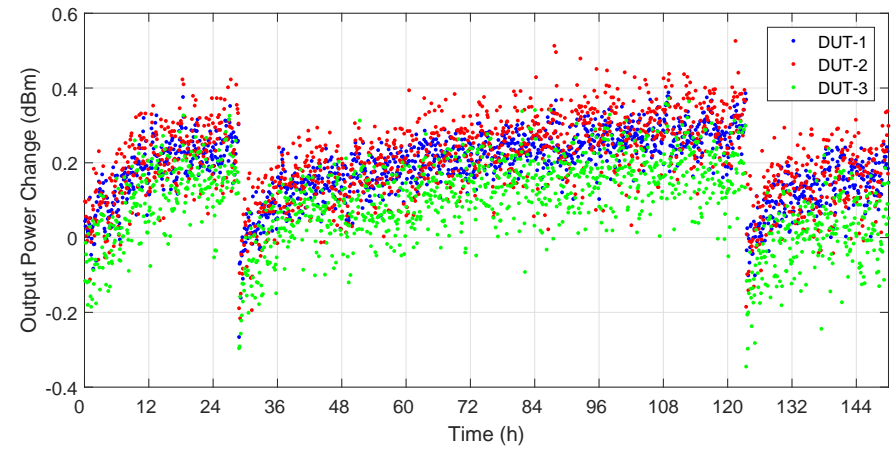

Figure 12. Change of output power over time. of RF circuits. Dependent on the test plan requirements, the concept can be easily scaled by increasing the number of multiplexer channels for testing of bigger batches or simultaneous testing of different devices during the same irradiation run. The proposed setup is not limited to the requirements of CHARM facility and may applied for other tests facilities as well.

\section{REFERENCES}

[1] Single Event Effects Test Method and Guidelines, European Space Agency Std. ESCC 25 100, Rev. 2, 2014.

[2] Total Dose Steady-State Irradiation Test Method, European Space Agency Std. ESCC 22 900, Rev. 4, 2010.

[3] Standard Guide for the Measurement of Single Event Phenomena (SEP) Induced by Heavy Ion Irradiation of Semiconductor Devices, American Society for Testing and Materials Std. F1192-11, 2011.

[4] Test Method Standard Microcircuits, Department of Defense Std. MILSTD-883J, 2015.

[5] J. Mekki, M. Brugger, R. G. Alía, A. Thornton, N. C. Dos Santos Mota, and S. Danzeca, "CHARM: A mixed field facility at CERN for radiation tests in ground, atmospheric, space and accelerator representative environments," IEEE Transactions on Nuclear Science, vol. 63, no. 4, pp. 2106-2114, 2016

[6] G. F. Engen and C. A. Hoer, "Thru-reflect-line: An improved technique for calibrating the dual six-port automatic network analyzer," IEEE Transactions on Microwave Theory and Techniques, vol. 27, no. 12, pp. 987-993, 1979.

[7] J. P. Dunsmore, "Transmission response measurement system and method of using time gating," Patent US7 170297 B1.

[8] S. Mueller, A. Och, S. Danzeca, R. G. Alia, M. Brugger, R. Weigel, and A. Koelpin, "Circuit design for a radiation tolerant $2.4 \mathrm{GHz}$ synthesizer based on COTS components," Topical Workshop on the Internet of Space (TWIOS), (submitted for publication). 